\title{
Sedation and Anesthesia of Pediatric Patient for External Beam Radiotherapy (XRT)
}

\author{
Ihab E. Afifi ${ }^{\text {a }}$, Ibrahim E. Mostafa ${ }^{\text {a }}$, Peter M. Saleh ${ }^{\text {b }}$
}

\begin{abstract}
a Department of anesthesia and ICU, faculty of medicine, Benha University, Egypt. ${ }^{\mathrm{b}}$ Department of Anesthesia, Matarya Teaching Hospital-Cairo, Egypt.
\end{abstract}

Correspondence to: Peter M. Saleh, Department of anesthesia and ICU, , Matarya Teaching Hospital-Cairo, Egypt.

Email:

petermelad25.pm@gmail.com

Received: 27 June 2020

Accepted: 22 March 2021

\begin{abstract}
:
Background: external beam radiation delivers a beam of highenergy $\mathrm{x}$-rays to a patient's tumor site, in order to destroy the cancer cells. external beam radiation gets its name from the fact that the beams come from an external source (a machine called a linear accelerator) and are aimed at the site of the tumor. Aim: was to obtain the most recent guideline in sedation and anesthesia of pediatric patient for external beam radiotherapy (xrt). providing sedation anesthesia techniques during the performance of diagnostic and therapeutic procedures on children decreases anxiety, discomfort, and pain and may improve child wellbeing. immobilization is a pre-requisite for accurate radiotherapy.
\end{abstract} Subjects and methods: this is a review article; the search was performed in medline, embase, pubmed, and cinhal. Results: anesthetic drugs can be used in sub-anesthetic doses to cause sedation but, because they are potent, the difference between sedative and anesthetic doses is too small and airway skills are required too often for nonanesthetists to use them. Conclusion: sedation and anesthesia of pediatric patients have serious associated risks, as hypoventilation, apnea airway obstruction and cardiopulmonary impairment or depression. these risks should be avoided, diagnosed rapidly and treated appropriately. medical practitioners responsible for the sedation process should know the nature of the sedatives and their side-effects. they should be able to detect and manage complications.this study was approved by the institutional ethical committee.

keywords: sedation; anesthesia; pediatric; external radiotherapy 



\section{Introduction}

Cancer continues to be a leading cause of pediatric mortality in the world, with physicians and scientists constantly developing new weapons to combat it. Chemotherapy, surgery, nutrition, and holistic medicine all have a place in the multimodal approach that can prolong longevity and ameliorate quality of life. As part of this armamentarium, external beam radiation therapy (XRT) has proven to be a safe and effective technique for the management of various malignant (and occasionally nonmalignant) lesions (1).

External beam radiation delivers a beam of high-energy X-rays to a patient's tumor site, in order to destroy the cancer cells. External beam radiation gets its name from the fact that the beams come from an external source (a machine called a linear accelerator) and are aimed at the site of the tumor. Providing sedation anesthesia techniques during the performance of diagnostic and therapeutic procedures on children decreases anxiety, discomfort, and pain and may improve child wellbeing. In addition, sedation anesthesia reduces the child's movement and thus may increase the success rate of the procedures required. As a result, over the last few years there has been increasing interest and considerable demand for sedation in the pediatric population (2).

For a successful procedure there must be a preparation for the patient, including adequate explanation on the procedure and risks should be given to the parent with appropriate reassurance of the patient to allay fear and anxiety, proper medical history, adequate fasting hours and applying simulation to ensure the precise dose and duration of sedative or anesthetic drugs (3).

Immobilization is a pre-requisite for accurate radiotherapy. Most children older than three to four years of age can be encouraged to lie still with good radiotherapy play specialist input. Anesthesia is required for younger children, and some older patients with learning difficulties or behavioral problems, and is more likely to be needed if beamdirecting shells or prone positioning are used. Although more than a dozen different professional organizations have issued guidelines indicating the optimal approach to monitoring sedation used í children undergoing painful procedures or diagnostic imaging, an observational study shows a "large degree of variability"in the monitoring that occurs in the real world, with differences 
between the types of sedation providers, medications, procedures, and patients (4).

Delivery of safe and effective anesthesia for pediatric oncology patients receiving radiation therapy presents several challenges. The procedure is usually brief, and the patient must remain motionless and often prone. Further, the anesthesia provider must continuously monitor the patient's cardiovascular and respiratory status from a remote location (5).

Aim of the work was to obtain the most recent guideline in sedation and anesthesia of pediatric patient for External beam radiotherapy (XBR).

\section{Material and Methods:}

This is a review article, the search was performed in MEDLINE, Embase, Pubmed and CINAHL Plus in the same date range with the following mediacl terms: "Sedation; anesthesia; pediatric; External beam radiotherapy.", including articles from 2000 to 2019. Excluded articles from review are those of langauge other than English. Key words: Sedation; anesthesia; pediatric; External radiotherapy.

\section{Results and discussion}

\section{Fundamentals of External Beam Radiotherapy (EBRT)}

Along with surgery, chemotherapy and nutrition, radiation therapy is a cornerstone in the multimodal management of pediatric cancer. Children with brain tumors, bone and soft tissues sarcomas, neuroblastoma, Wilms tumor, and Hodgkin lymphoma are commonly treated with radiation therapy. Advances in radiation therapy and the newer modality of proton beam therapy have enabled radiation oncologists to target tumors more successfully, plus avoiding damage to normal tissues. Anesthesiologists are increasingly being asked to provide sedation or anesthesia to ensure a completely immobile patient during radiation therapy (6).

External beam radiotherapy destroys cancer cells using radiation aimed at a cancer from a machine. The types of radiation used include high energy photon beams, or particle beams, such as protons, electrons or neutrons. The most common types of external radiotherapy use photon beams (either as X-rays or gamma rays). The radiotherapy beams destroy the cancer cells in the treatment area (7). 


\section{Types of EBRT}

\section{A. Standard (Conventional) fractionation}

The most common schedule for external beam radiation divides the total dose of radiation into several smaller doses or fractions. Treatments are usually given once a day, 5 days a week, and may last for several (about 3-8) weeks (8).

\section{1) Hyperfractionation}

In this type, the daily dose of radiation is given over 2 or more sessions each day. The total period of time to complete the treatment schedule is not changed. By using multiple smaller doses of radiation, a higher overall dose can be given (8).

\section{2) Accelerated fractionation}

With accelerated radiation therapy, the total dose of radiation is given over a shorter period of time by giving the same dose of radiation more than once a day. Accelerated fractionation does not change the total radiation dose (8).

\section{3) Hypofractionation}

With hypofractionated radiation therapy, fewer radiation treatments are given. This is done by giving either a short course of daily treatments or by giving fewer large doses (sometimes just a single treatment) (9).

\section{B. Conformal radiation therapy}

Conformal radiation therapy is a type of external beam radiation therapy. For this procedure, the same type of machine used for regular radiotherapy treatment is combined with a specialized device called a multi-leaf collimator (8).

\section{Intensity-modulated radiation therapy (IMRT)}

It is a type of external beam radiation that uses computer-controlled radiation beams in conjunction with three-dimensional computed tomography images of the tumor site and surrounding area. IMRT delivers targeted radiation doses to the tumor site, patterned to match the shape of the tumor through modulating the intensity of the radiation beams. The beam strength varies across the treatment area. The tumor receives radiation from a series of small beams of different strengths. IMRT shapes the treatment beams very precisely and allows the dose of radiation to be adjusted for different parts of the treatment area. This technology not only means that the tumor site receives the high doses needed to destroy cancer cells, it also spares surrounding organs and tissue (10).

D. Image-guided radiation therapy (IGRT) 
IGRT uses imaging scans and X-rays to make sure the radiotherapy treatment is as accurate as possible. During or before treatment, $\mathrm{X}$ rays and scans (2D or 3D images) are available to make sure that the beams are aimed at the correct place. This helps reduce the dose of radiation given to the healthy tissue around the tumor and reduces side effects. Some people have small markers put into the area of the tumor. The markers show up on X-ray and allow the radiographer to target the radiotherapy accurately (11).

\section{E. Stereotactic radiation therapy}

Stereotactic radiation therapy is a type of external beam radiation therapy. Stereo means three-dimensional (3-D) and tactic means to probe. Stereotactic radiation therapy allows radiation beams to be given to a very specific area, usually the brain. It is mainly used to treat small brain tumors that are either malignant or benign (12).

\section{F. Proton beam radiotherapy}

Protons are nuclei of hydrogen atoms accelerated by a cyclotron or synchrotron. Protons have different characteristics from conventional photon treatment (e.g., gamma or X-rays); proton therapy is associated with significant benefits over photons in terms of normal tissue sparing and potential radiation dose escalation for many patients with malignancies. For these qualities, the availability of this technology is increasing rapidly. The physical properties of protons allow achievement of a very important benefit which is decrease in radiation delivered outside of the target volume (13).

\section{G. Neutron beam therapy}

Like proton therapy, neutron beam therapy is a specialized form of external beam radiation therapy. It is often used to treat certain tumors that are radioresistant, meaning that they are very difficult to be destroyed using conventional X-ray radiation therapy. Neutrons have a greater biologic impact on cells than other types of radiation. Used carefully, this added impact can be an advantage in certain situations. Neutron therapy is available at only a few specialized centers (14).

\section{Pre-Procedural Preparation}

\section{a.Pre-Procedural Evaluation of the Patient:}

Evaluation of the child's health before sedation should be performed to detect possible interval changes between every sessions . This provides an opportunity to identify specific risk factors and may warrant additional consultation before sedation. Health evaluation will also screen out patients 
whose sedation will require more advanced airway or cardiovascular management skills or alteration in the doses or types of medications used for procedural sedation (15).

The aspiration risk for procedural sedation and analgesia is lower than that of general anesthesia because the principal risk factors (airway manipulation, absence of protective airway reflexes and poor ASA physical status) are not present routinely in this setting. As a reflection of this evidence, some emergency physicians disregard preprocedural fasting guidelines. However, even though some published studies suggest that strict adherence to the fasting guidelines is not necessary, their sample size and/or designs are insufficient to safely practice the liberalized preprocedural fasting guidelines and to justify changes in emergency department procedural sedation and analgesia policies (15).

Children receiving deep sedation should have an intravenous access placed at the start of the procedure. An intraosseous needle should be available in the event of failure to place an intravenous line, or if the intravenous line becomes nonfunctional in an emergency situation. If the child is receiving sedative agents other than via the intravenous route; for example, intranasal, oral or rectal, the need for intravenous access is debatable. Most authors recommend the placement of intravenous access for administration of emergency medications, including reversal agents, during procedural sedation. Sometimes, children having radiation therapy have central venous access in place. It is important to follow strict aseptic technique when accessing these lines and handling drugs to prevent central line-associated bloodstream infection. Dehydrated children may require preprocedural fluid administration (15).

\section{b. SpecificPreparations for Sedation and Analgesia:}

Part of the safety net of sedation is to use a systematic approach (Table 1) so as not to overlook having an important drug or a piece of equipment immediately available at the time of a developing emergency(16). 
Table (1):SOAPME is a commonly used acronym that is useful in preparation for a procedural sedation and analgesia(16).

S (suction)

O (oxygen)

A (airway)

P (pharmacy)

M (monitors)

E (equipment)
Size-appropriate suction catheters and a functioning suction apparatus.

adequate oxygen supply and functioning flowmeters/other devices to allow its delivery.

size-appropriate airway equipment [nasopharyngeal and oropharyngeal airways, laryngoscope blades (checked and functioning), endotracheal tubes, stylets, face mask, bag-valve-mask or equivalent device (functioning)].

all the basic drugs needed to support life during an emergency, including antagonists as indicated.

functioning pulse oximeter with size-appropriate oximeter probes and other monitors as appropriate for the procedure [ e.g.,non-invasive blood pressure and end-tidal carbon dioxide monitors, electrocardiography (ECG) machines, stethoscopes].

special equipment or drugs for a particular case (e.g.,defibrillator).

\section{Definitions, Classification and Techniques of Sedation}

The American College of Emergency Physicians (ACEP) defines procedural sedation as "a technique of administering sedatives or dissociative agents with or without analgesics to induce a state that allows the patient to tolerate unpleasant procedures while maintaining cardiorespiratory function". Procedural sedation and analgesia (PSA) is intended to result in a depressed level of consciousness that allows the patient to maintain oxygenation and airway control independent (17).

Another definition of sedation is that it is the depression of the central nervous system and/or reflexes by administration of drugs to decrease patient discomfort without producing unintended loss of consciousness (18).

\section{Levels of sedation}

The level of sedation may be:

\section{A. Minimal sedation and anxiolysis}

A drug-induced state during which patients are awake, calm and respond normally to verbal commands. Cognitive function and coordination might be impaired, ventilatory and cardiovascular functions are unaffected (19).

\section{B. Moderate sedation and analgesia}

Drug-induced depression of consciousness during which patients are sleepy but respond purposefully to verbal commands or light tactile stimulation (reflex withdrawal from a painful stimulus is not a purposeful 
response). No interventions are required to maintain a patent airway. Spontaneous ventilation is adequate. Cardiovascular function is usually maintained. Some healthcare practitioners regard conscious and moderate sedation as synonymous (16).

\section{Deep sedation and analgesia}

Drug-induced depression of consciousness during which patients are asleep and cannot be easily aroused but do respond purposefully to repeated or painful stimulation. The ability to maintain ventilatory function independently may be impaired. Patients may require assistance to maintain a patent airway. Spontaneous ventilation may be inadequate. Cardiovascular function is usually maintained (16).

\section{Classification of sedation}

Some authors classify sedation according to the type into:

\section{A. Non-dissociative sedation}

Non-dissociative sedative drugs (including opioids, benzodiazepines, barbiturates, etomidate and propofol) operate on the sedation dose-response continuum. Higher doses provide progressively deeper levels of sedation with possible respiratory and cardiovascular compromise, central nervous system depression, and unconsciousness. With the use of non-dissociative drugs, the key to minimizing adverse events is the careful titration of drugs to the desired effect (20).

\section{B. Dissociative sedation:}

Dissociative sedation produced by ketamine, causes a trance-like cataleptic state characterized by intense analgesia, amnesia, sedation, retention of protective reflexes (as deeper levels of sedation are reached, airway reflexes may be obtunded), spontaneous breathing and cardiovascular stability. It is believed that, when ketamine is administered in dissociative doses, it does not operate on the sedation continuum (20).

\section{$\underline{\text { Sedation techniques }}$}

Sedation should have a rapid, smooth onset and recovery without adverse effects; such as nausea and vomiting, which may delay the discharge of patients (21).

\section{A. Simple/basic sedation}

Simple/basic sedation is induced by a single agent and not a combination of agents; for example, oral, transmucosal or rectal drugs such as a small dose of an oral benzodiazepine or inhalation of nitrous 

oxide (N2O) in at least $50 \%$ oxygen. Sedation can no longer be considered simple or basic once additional agents become necessary and the depth of sedation may not be advanced unless the patient is fasting (20).

\section{B. Advanced sedation}

Advanced sedation is induced by one of the following techniques; any combination of drugs administered by any route, any sedation administered by the intravenous route using bolus or infusion techniques, or any inhalational sedation (e.g., sevoflurane) with the exception of $\mathrm{N} 2 \mathrm{O}$ used as the sole agent in a concentration of less than $50 \%$ in oxygen. Advanced sedation can include both dissociative and non- dissociative techniques (20).

\section{Anesthetic Management}

Pediatric anesthesiologists commonly provide anesthesia or sedation outside the operating room. These locations include MRI, CT, and nuclear medicine scanners; interventional radiology and gastrointestinal laboratories; cardiac catheter and electrophysiology laboratories; and hematology/2oncology procedure rooms (22). Although they are perhaps more at home working outside the 'comfort zone' of the operating room than adult anesthesiologists, there are unique considerations for providing safe anesthesia in a radiation therapy suite (22).

Also, children requiring radiation therapy are often very sick, sometimes even needing daily transfers from a pediatric ICU for their treatments. In addition, separation from the parents may be a problem in some cases. Behavior of the children may widely vary (Table 2) (23).

Table (2): Response to parent separation(23)

\begin{tabular}{lll}
\hline $\begin{array}{l}\text { Behavior of the child during separation } \\
\text { from parents }\end{array}$ & Criteria & Score \\
\hline Excellent & Patient unafraid, cooperative, or asleep & 1 \\
Good & Slightly feared/crying, quiet with reassurance & 2 \\
Fair & Moderately feared and crying, not quiet with reassurance & 3 \\
Poor & Crying, need for restraint & 4 \\
\hline
\end{tabular}



Children requiring radiation therapy can experience considerable anxiety. This is related to the unfamiliar and potentially frightening radiation equipment and immobilization devices, particularly the plastic immobilization cast of the head, which fits tightly over the face. Children have often undergone a range of procedures prior to their radiotherapy treatment, and may be suitably suspicious of strangers. The child has to be alone in the treatment vault during the treatments, and must lie completely still
In children older than age 7 or 8 years, it is often possible to perform radiotherapy without sedation. This may be accomplished in younger children with behavioral rehearsal and distraction techniques. Audiovisual interventions, picture books explaining the procedure, playful inclusion of toys, and a reward system using beads as tokens for every accomplished radiation therapy session have been used (24). (24).

Table (3): Commonly used drugs for procedural sedation and analgesia in pediatric patients. (25)

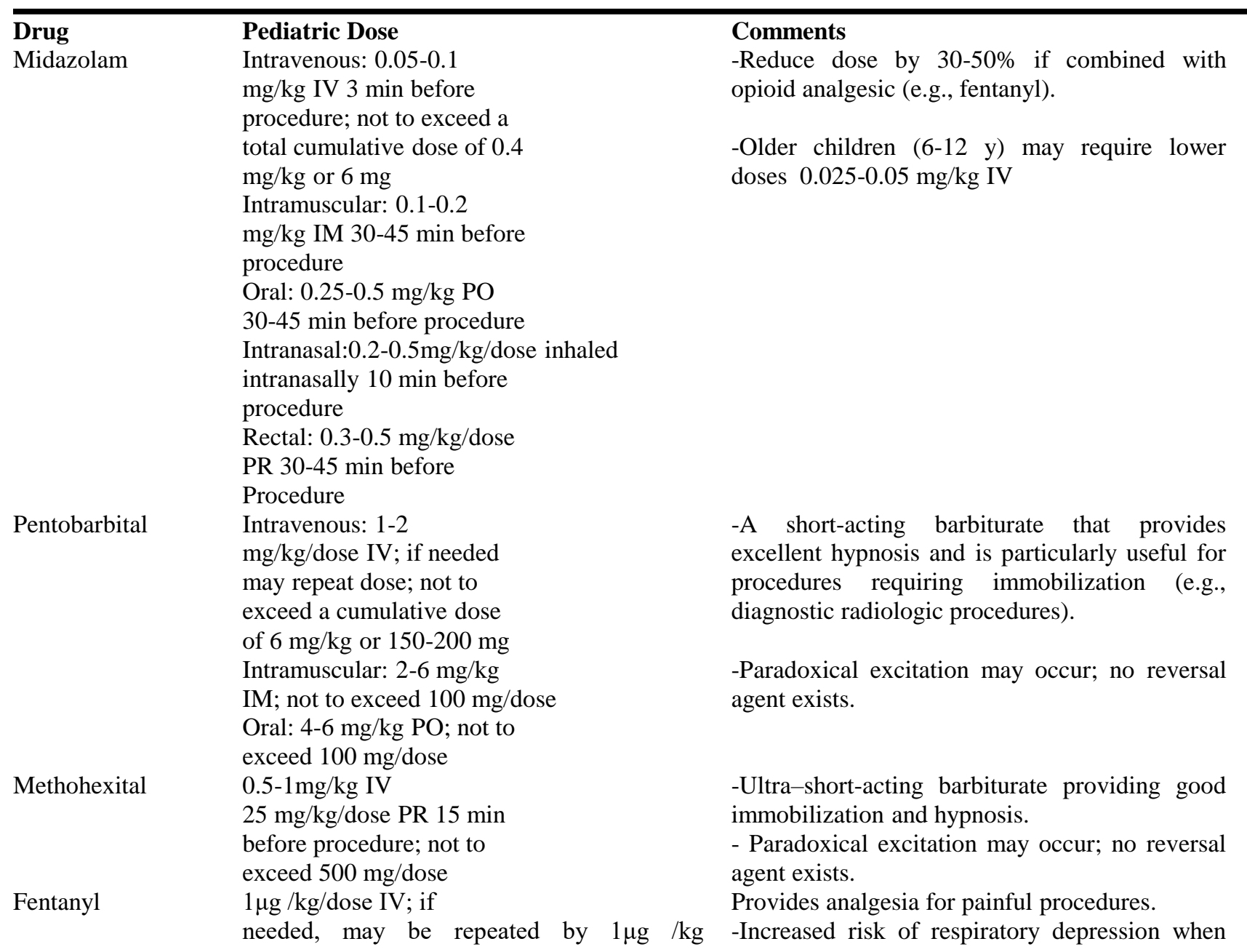




\section{Drug}

Propofol

Chloral

Hydrate

\section{Pediatric Dose}

increments; not to

exceed total cumulative

dose of $4 \mu \mathrm{g} / \mathrm{kg}$

Ketamine

Intravenous: $1-2 \mathrm{mg} / \mathrm{kg}$

loading dose IV; $0.25-1$ slowly, not to exceed $0.5 \mathrm{mg} / \mathrm{kg} / \mathrm{min}$

Intramuscular: 2-5

$\mathrm{mg} / \mathrm{kg} / \mathrm{dose}$ IM

mixed in cola or other

beverage $30 \mathrm{~min}$ before procedure $\mathrm{mg} / \mathrm{kg}$ IV every 10-15min; administer

Oral: 6-10 mg/kg/dose PO

Data limited: $1-1.5 \mathrm{mg} / \mathrm{kg}$

IV loading dose; 0.25-0.5

$\mathrm{mg} / \mathrm{kg}$ IV every $3-5 \mathrm{~min}$ or $50-150 \mu \mathrm{g}$

$/ \mathrm{kg} /$ min continuous IV infusion

$50-75 \mathrm{mg} / \mathrm{kg} / \mathrm{dose} \mathrm{PO} / \mathrm{PR}$;

not to exceed $1 \mathrm{~g} / \mathrm{dose}$

(infants) or $2 \mathrm{~g} / \mathrm{dose}$

(children); administered 30

min before procedure

\section{Comments}

combined with sedatives (reduce sedative dose).

-Chest wall rigidity associated with rapid IV push.

-Provides excellent sedation and analgesia.

- Elicits dissociative state.

- Increases bronchial and salivary

secretions.

-Increases heart rate, blood pressure, and intracranial pressure. -Emergence

hallucinations observed

inolder children (>15 years) and adults.

-Pharmacologic effects NOT reversible.

-Provides rapid anesthesia.

-Apnea occurs upon induction and unpredictably causes loss of airway reflexes (even at sedative doses).

-Irritation and burning with IV

administration.

-Effect NOT reversible

-No longer recommended since much safer and more effective alternatives exist.

-Unpredictable effect; paradoxical hyperactivity may occur.

-May cause nausea and vomiting. -Decrease dose if combined with opioid analgesic (e.g., fentanyl).

-Deaths or permanent neurologic injury from respiratory compromise have been reported, particularly in those with risk factors (e.g., ASA class III, Leigh encephalopathy, tonsillar and adenoidal hypertrophy, obstructive sleep apnea). -Active metabolite has prolonged half-life.

Min $=$ minutes

$\mathrm{PO}=$ per orum

$\mathrm{PR}=$ per rectum

\section{4- Monitoring}

The most precious periods in sedation or general anesthesia are the 5-10 minutes after IV administration of medication and during the period immediately after the end of the procedure when procedural stimuli are ceased. Therefore, during sedation or general anesthesia, the following parameters should be continuously monitored: the level of consciousness every 15 minutes (when possible) using the pediatric Glasgow coma scale and the Ramsay sedation scale (16). A sedation score specific for the pediatric age group is available (23). 
It is important for anesthesiologists to understand potential complications that pediatric patients may experience when undergoing anesthesia for repetitive treatments. There are many factors that influence post-treatment side effects and complications, including cancer type/location, receiving chemotherapy, type of anesthetic used, duration and degree of sedation, positioning and airway access, and other medical conditions. Understanding of these factors and their potential complications allows for more precise and complete radiotherapuetic management of patients(26).

\section{5- Criteria for Discharge}

One of the requirements of patient assessment and care after sedation is to evaluate the patient's recovery as the sedation wears off. This is very important in the post-anesthesia care unit (PACU) after a procedure with general anesthesia. The modified Aldrete scale has been very widely used by the anesthesiologists (Table 4). It is utilized to decide when the patient is "recovered" enough to move through the phases of PACU, and ultimately to go home or back to their room (27)

Table (4): The 'modified"Aldrete scale, (27).

\begin{tabular}{|c|c|c|c|}
\hline Score & 2 & 1 & $\mathbf{0}$ \\
\hline Respiration & $\begin{array}{l}\text { Able to take deep breath } \\
\text { and cough }\end{array}$ & Dyspnea/Shallow breathing & Apnea \\
\hline $\mathrm{O}_{2}$ saturation & $\begin{array}{l}\text { Maintains } \geq 92 \% \text { on room } \\
\text { air }\end{array}$ & $\begin{array}{l}\text { Needs } \mathrm{O}_{2} \text { inhalation to } \\
\text { maintain } \\
>90 \%\end{array}$ & $\begin{array}{l}\text { Saturation }<90 \% \text { even with } \\
\text { supplemental } \mathrm{O}_{2}\end{array}$ \\
\hline Consciousness & Fully awake & Arousable or calling & Not responding \\
\hline Circulation & $\mathrm{BP} \pm 20 \mathrm{mmHg}$ preop. & $\mathrm{BP} \pm 20-50 \mathrm{mmHg}$ preop. & $\mathrm{BP} \pm 50 \mathrm{mmHg}$ preop. \\
\hline Activity & $\begin{array}{l}\text { Able to move } 4 \\
\text { extremities voluntarily or } \\
\text { on command }\end{array}$ & $\begin{array}{l}\text { Able to move } 2 \text { extremities } \\
\text { voluntarily }\end{array}$ & $\begin{array}{l}\text { Able to move } 0 \text { extremities } \\
\text { voluntarily }\end{array}$ \\
\hline
\end{tabular}

Preop. $=$ compared to the preoperative value 


\section{Conclusion}

Anesthetic drugs can be used in subanesthetic doses to cause sedation but, because they are potent, the difference between sedative and anesthetic doses is too small and airway skills are required too often for non-anesthetists to use them. Conversely, safe sedation drugs are not as potent, have a wide margin of safety and will not always succeed. Increasing doses increases success but some chíldren will sleep too deeply. Sedation drugs also have a long action and are unpredictable even standard doses can cause deep and prolonged sedation.Occasionally, there are 'paradoxical' distress reactions perhaps attributable to dizziness, anxiety or hallucinations. Sedation and anesthesia of pediatric patients have serious associated risks, as hypoventilation, apnea airway obstruction and cardiopulmonary impairment or depression. These risks should be avoided, diagnosed rapidly and treated appropriately. Medical practitioners responsible for the sedation process should know the nature of the sedatives and their side-effects. They should be able to detect and manage complications.

\section{References}

1. Harris EA. Sedation and anesthesia options for pediatric patients in the radiation oncology suite. Int J Pediatr. 2010;2010.
2. Gozal D, Drenger B, Levin PD, Kadari A, Gozal Y. A pediatric sedation/anesthesia program with dedicated care by anesthesiologists and nurses for procedures outside the operating room. J Pediatr. 2004;145(1):47-52.

3. Scottish IGN. SIGN Guideline 58: safe sedation of children undergoing diagnostic and therapeutic procedures. Paediatr Anaesth. 2008;18(1):11.

4. McMullen KP, Hanson T, Bratton J, Johnstone PAS. Parameters of anesthesia/sedation in children receiving radiotherapy. Radiat Oncol. 2015;10(1):65.

5. Anghelescu DL, Burgoyne LL, Liu W, Hankins GM, Cheng C, Beckham PA, et al. Safe anesthesia for radiotherapy in pediatric oncology: St. Jude Children's Research Hospital experience, 20042006. Int J Radiat Oncol Biol Phys. 2008;71(2):491-7.

6. Sheer D, Jeyapalan J, Doctor GT, Jones TA, Alberman SN, Tep A, et al. DNA Methylation Analysis of Paediatric Low-Grade Astrocytomas Identifies a Tumour-Specific Signature at a Set of Enhancers. Pediatr Blood Cancer. 2015;

7. Baskar R, Lee KA, Yeo R, Yeoh K-W. Cancer and radiation therapy: current advances and future directions. Int J Med Sci. 2012;9(3):193.

8. Chowdhury MR, Schumann C, Bhakta-Guha D, Guha G. Cancer nanotheranostics: Strategies, promises and impediments. Biomed Pharmacother. 2016;84:291-304.

9. Vanaki Z, Matourypour P, Gholami R, Zare Z, Mehrzad V, Dehghan M. Therapeutic touch for nausea in breast cancer patients receiving chemotherapy: Composing a treatment. Complement Ther Clin Pract. 2016;22:64-8.

10. Ramzy L, Nasr M, Metwally AA, Awad GAS. Cancer nanotheranostics: A review of the role of conjugated ligands for overexpressed receptors. Eur J Pharm Sci. 2017;104:273-92.

11. Zhao W, Han B, Yang Y, Buyyounouski M, Hancock SL, Bagshaw $\mathrm{H}$, et al. Incorporating imaging information from deep neural network 
layers into image guided radiation therapy (IGRT). Radiother Oncol. 2019;140:167-74.

12. Kun LE. General principles of radiation oncology. Princ Pract Pediatr Oncol 6th Ed Philadelphia, PA Wolters Kluwer/Lippincott Williams Wilkins Heal. 2011;406-25.

13. Hill-Kayser CE, Both S. Proton therapy: ever shifting sands and the opportunities and obligations within. Front Oncol. 2011;1:24.

14. Davis C, Sikes J, Namaranian P, Laramore G, Dillon JK. Neutron beam radiation therapy: an overview of treatment and oral complications when treating salivary gland malignancies. J Oral Maxillofac Surg. 2016;74(4):830-5.

15. Ramaiah R, Bhananker S, Krauss, Krauss, Malviya, Cote, et al. Pediatric procedural sedation and analgesia outside the operating room: anticipating, avoiding and managing complications. Expert Rev Neurother. 2011;11(5):755-63.

16. Arlachov $\mathrm{Y}$, Ganatra RH. Sedation/anaesthesia in paediatric radiology. $\mathrm{Br} \mathrm{J}$ Radiol. 2012;85(1019):e1018-31.

17. Godwin SA, Burton JH, Gerardo CJ, Hatten BW, Mace SE, Silvers SM, et al. Clinical policy: procedural sedation and analgesia in the emergency department. Ann Emerg Med. 2014;63(2):247-58.

18. Loepke AW, Davidson AJ. Surgery, Anesthesia, and the Immature Brain. In: A Practice of Anesthesia for Infants and Children. Elsevier; 2019. p. 581-603.

19. FALLAH R, FERDOSIAN F, SHAJARI A. Non-Parenteral Medications for Procedural Sedation in Children-A Narrative. Iran $\mathbf{J}$ child
Neurol. 2015;9(3):1.

20. Hinkelbein J, Lamperti M, Akeson J, Santos J, Costa J, De Robertis E, et al. European Society of Anaesthesiology and European Board of Anaesthesiology guidelines for procedural sedation and analgesia in adults. Eur $\mathrm{J}$ Anaesthesiol. 2018;35(1):6-24.

21. Tobias JD, Leder M. Procedural sedation: A review of sedative agents, monitoring, and management of complications. Saudi J Anaesth. 2011;5(4):395.

22. McFadyen JG, Pelly N, Orr RJ. Sedation and anesthesia for the pediatric patient undergoing radiation therapy. Curr Opin Anesthesiol. 2011;24(4):433-8.

23. Bhadla S, Prajapati D, Louis T, Puri G, Panchal S, Bhuva M. Comparison between dexmedetomidine and midazolam premedication in pediatric patients undergoing ophthalmic day-care surgeries. Anesth essays Res. 2013;7(2):248.

24. Brown NW. Psychoeducational groups: Process and practice. Routledge; 2018.

25. Bocskai T, Németh A, Bogár L, Pytel J. Sedation of children for auditory brainstem response using ketamine-midazolam-atropine combination-a retrospective analysis. Springerplus. 2013;2(1):178.

26. Verma V, Beethe AB, LeRiger M, Kulkarni RR, Zhang M, Lin C. Anesthesia complications of pediatric radiation therapy. Pract Radiat Oncol. 2016;6(3):143-54.

27. Dowling LP. Aldrete Discharge Scoring: Appropriate for Post Anesthesia Phase I Discharge? 2015;

To cite this article: Ihab E. Afifi, Ibrahim E. Mostafa, Peter M. Saleh. Sedation and Anesthesia of Pediatric Patient for External Beam Radiotherapy (XRT). BMFJ 2021; 38(1):234-246, DOI: 10.21608/ bmfj.2021 .33194.1281 